\title{
One-Dimension Nonisentropic Model for the Flow of Aluminized Explosive Products
}

\author{
Ji Duan, ${ }^{1}$ Cheng Wu, ${ }^{2}$ Fengjiang An, ${ }^{2}$ Shasha Liao, ${ }^{2}$ Hengzhu Liu, ${ }^{2}$ and Lihui Dai ${ }^{2}$ \\ ${ }^{1}$ College of Electron-Mechanics Engineering, Beijing Institute of Technology, Beijing 100081, China \\ ${ }^{2}$ State Key Laboratory of Explosion Science and Technology, Beijing Institute of Technology, Beijing 100081, China \\ Correspondence should be addressed to Cheng Wu; chengwu@bit.edu.cn
}

Received 3 January 2017; Accepted 22 February 2017; Published 4 April 2017

Academic Editor: Babak Shotorban

Copyright (c) 2017 Ji Duan et al. This is an open access article distributed under the Creative Commons Attribution License, which permits unrestricted use, distribution, and reproduction in any medium, provided the original work is properly cited.

\begin{abstract}
A new analytic model of aluminized explosive products based on the method of characteristics for planar isentropic flow is proposed herein. The contribution of $\mathrm{Al}$ oxidation in the explosion products is investigated analytically. The flow behind the detonation front cannot be treated as isentropic due to the $\mathrm{Al}$ oxidation in the products. To solve the nonisentropic flow field of aluminized explosives products analytically, the assumption of local isentropic process is proposed. Based on this assumption, the flow field behind the detonation front of aluminized explosive is a function of only the reacted aluminum mass fraction in each time range. The metal plate test was conducted with the metal plate driven by RDX/Al/wax (76/20/4) and RDX/LiF/wax (76/20/4). The reacted aluminum mass can be obtained indirectly from the experiment results. The reacted aluminum mass was then applied to the analytic model, and the velocity of metal plate driven by RDX/Al/wax (76/20/4) and RDX/LiF/wax (76/20/4) was calculated. The final velocity of the metal plate driven by $\mathrm{RDX} / \mathrm{Al} /$ wax was $7.8 \%$ higher than that driven by $\mathrm{RDX} / \mathrm{LiF} /$ wax.
\end{abstract}

\section{Introduction}

The addition of aluminum to condensed explosives to increase the total energy release of the explosive is common practice. Aluminum in its powdered form generally does not react quickly enough to contribute to the detonation front itself [1]; however, it can react in the products of the condensed phase explosive or in the surrounding atmosphere, significantly contributing to the strength and acceleration of the blast wave.

Often, a multiphase computational fluid dynamics (CFD) model is used (Frost et al. [2, 3], Zhang et al. [4], Ripley et al. [5], Milne et al. [6], Cooper et al. [7], Massoni et al. [8], Kim et al. [9], etc.) to predict the conditions for metal particle reaction in explosives. Mesoscale modeling with hydrocodes is also widely used (Frost et al. [10], Ripley et al. [11], Zhang et al. [12], Milne et al. [6], etc.). Although these models can, in principle, describe a great amount of detail about the phenomena of interest, developing an analytic model that captures the key elements of the problem in a way that makes the dominant features easily discernible would be preferred. Just like the classical Seshadri formulation [13] analytically describes the structure of premixed flames propagating in a uniform cloud of fuel particles. To establish the analytic model, the author simplified the problem and made some assumptions (gravitational effects, diffusion caused by pressure gradient, and heat transport by radiation is negligible). The Seshadri formulation is used to analyze the flame propagating under normal pressure and temperature. It is not suitable to analyze the combustion of $\mathrm{Al}$ and detonation products under high pressure and temperature. In this paper, we have developed a new model based on the classical theory for ideal explosive products (Taylor [14]) that incorporates the oxidation of $\mathrm{Al}$ in the products, allowing us to analytically investigate the contribution of $\mathrm{Al}$ oxidation in the detonation product.

The flow behind the detonation front of the aluminized explosive is more complex than the flow behind the detonation front of an ideal explosive. To analytically solve the flow behind the detonation front of the aluminized explosive, it is necessary to make some assumptions. For example, we propose an assumption called local isentropic process, which enables the conclusion that the flow field behind the 
detonation front of aluminized explosive is only a function of the reacted aluminum mass fraction at each time. A metal plate test was conducted to obtain the velocity of the plate and, indirectly, the mass fraction of reacted aluminum powder. Applying the model and the described assumptions, we calculated the velocity of metal plate driven by the $\mathrm{RDX} / \mathrm{Al} /$ wax $(76 / 20 / 4)$ and $\mathrm{RDX} / \mathrm{LiF} /$ wax $(76 / 20 / 4)$. The final velocity driven by $\mathrm{RDX} / \mathrm{Al} /$ wax is $7.8 \%$ higher than the final velocity driven by $\mathrm{RDX} / \mathrm{LiF} /$ wax. We then compared the test result with the result calculated by our analytic model and found that the final velocity of metal plate driven by the $\mathrm{RDX} / \mathrm{Al} /$ wax $(76 / 20 / 4)$ calculated by the analytic model is $4.5 \%$ higher than the test result.

\section{The Change of Entropy of the Detonation Products}

The flow of the expanding detonation products is in general a highly complex problem. In the classic theory for the flow of detonation products, it is assumed that the flow behind the detonation front of explosive contained in a tube strong enough to confine the motion to one dimension and detonation products expand in a vacuum with $\gamma=3$ (Stanyukovich [15]). The flow of detonation products is isentropic approximately. As a result, the flow of the expanding detonation products can be solved analytically. Due to the reaction of $\mathrm{Al}$ particles with the products, however, the classic theory for the flow of detonation products is not suitable for the nonisentropic flow of detonation products of aluminized explosive. In this section, the change of entropy of the detonation products caused by chemical reaction will be discussed.

In the present study, it is assumed that the flow of detonation products in the tube is adiabatic. In the second law of thermodynamics, entropy is an extensive state function under a reversible process: $d S=d Q / T$. However, the expansion of the detonation products with chemical reactions is irreversible process; thus

$$
d S>\frac{d Q}{T}>0,
$$

where $Q$ is the heat released by chemical reaction and $T$ is the temperature of the products.

According to (1), the entropy of detonation products behind the detonation front increases with mass fraction of reacted aluminum powder. Actually, the change of entropy caused by the chemical reaction contains two parts: entropy flow and entropy generation. The entropy flow is caused by the change of heat in the thermodynamic system and the entropy generation is caused by the irreversible factors. In this section, the heat released by chemical reaction is the main reason for the change of entropy in the products. So the entropy generation caused by the irreversible factors will not be discussed. In order to model the flow behind the detonation front of the aluminized explosive, the contribution of the $\mathrm{Al}$ oxidation in the products must be considered. However, the flow of detonation products of aluminized explosive is more complex than the flow of detonation products of ideal explosive. Some assumptions are necessary to solve the model analytically and will be discussed in the next section.

\section{The Assumption of Analytic Model}

To solve the flow behind the detonation front and the contribution of $\mathrm{Al}$ oxidation in the products analytically, we simplified the problem. Consider the detonation of aluminized explosive contained in a tube so strong that the motion is confined to one dimension. The flow field generated by the high-explosive detonation products is modeled in a highly idealized manner: the products are treated as a perfect gas expanding into vacuum, with planar geometry. This simplification is based on the classical one-dimension model for ideal explosive. The Al particles are uniformly distributed in the products.

3.1. The Assumption of Al Particles in Detonation Products. In this paper, it is assumed that none of the aluminum particles reacted during the detonation and the $\mathrm{Al}$ oxidation occurs in the products behind the detonation front [16, 17]. For detonation of aluminized explosives, the duration for energy release of the reaction of explosive components is generally less than $0.1 \mu \mathrm{s}$, while the energy release for $\mathrm{Al}$ oxidation is in the order of microseconds to several milliseconds $[18,19]$. Therefore, the reaction rate of explosive components is much quicker than the rate of $\mathrm{Al}$ oxidation. So the assumption mentioned above is valid for aluminized explosive.

It is assumed that $\mathrm{Al}$ particles in the flow field do not affect the flow before $\mathrm{Al}$ particles oxidation. This assumption is valid for the small particle loading in the high explosive (e.g., Rudinger [20]). The small particles could be viewed as small perturbation to the flow field of detonation products.

The afterburning of the aluminum is modeled following the work of [21], which was originally applied to the combustion of aluminum in a gas phase. We adapted the Noble-Abel equation to determine the EOS of gas phase aluminum (Kim et al. [22]).

3.2. The Assumption of Local Isentropic Process. The flow behind the detonation front of aluminized explosive cannot be treated as an isentropic process approximately due to the chemical reaction of $\mathrm{Al}$ particles in the detonation products. We propose an assumption of local isentropic process to analyze the nonisentropic flow behind the detonation front of aluminized explosive.

The rate of aluminum oxidation is relatively slow, so we divided the products expansion process into many small ranges along the time axis. In each short time range, we assume that the process is approximately isentropic (the mass fraction of reacted aluminum is approximately constant). Using this method simplifies the flow behind the detonation front of aluminized explosive and enables analytical evaluation. We call this assumption local isentropic process.

Note that the detonation of aluminized explosive is extremely violent and fast; it is difficult to obtain detailed quantitative data related to this phenomenon directly. In this 
paper, the comparison of the velocities determined experimentally and obtained through the model is conducted. The model correctly described the contribution of $\mathrm{Al}$ oxidation in the detonation products of aluminized explosive. The assumption of local isentropic process is indirectly validated by this result.

\section{Model of Aluminized Explosive Products}

In this study, it is assumed that the detonation of aluminized explosive contained in a tube is strong enough to confine the flow of the detonation products to one dimension. As mentioned in the assumption of aluminized explosive products, the high-explosive detonation products are treated as a perfect gas and the flow field generated by the highexplosive detonation products is not affected by $\mathrm{Al}$ particles before their oxidation. The state equation of high-explosive detonation products is

$$
P_{\text {explosive }}=b \rho R T,
$$

where $b$ is the initial high-explosive mass fraction of aluminized explosive.

It is also assumed that the $\mathrm{Al}$ particles are uniformly distributed in the explosive and the $\mathrm{Al}$ particles exist in gas phase in the products. To determine the EOS of gas phase aluminum, we adapted the Noble-Abel equation of the form [22]

$$
P_{\mathrm{Al}}=\frac{a \lambda_{\mathrm{Al}} \rho R T}{1-n A_{n}}\left(n A_{n}: \sim 0.75\right),
$$

where $R$ denotes gas constant, $\lambda_{\mathrm{Al}}$ is the reacted aluminum mass fraction, $\rho$ is the density of aluminized explosive products, $a$ is the initial aluminum mass fraction of aluminized explosive, $n$ is the number of moles per unit volume, and $A_{n}$ is an empirical constant. The value of $n A_{n}$ is obtained from [22], due to the fact that aluminized explosive studied in [22] is similar to our study. (Note that the influence of value of $n A_{n}$ on our model is weak.)

The standard mixture rule applies to the aluminized explosive products. The state equation of aluminized explosive products is

$$
\begin{aligned}
P_{\text {total }} & =P_{\text {explosive }}+P_{\mathrm{Al}}=\left(b+a \frac{\lambda_{\mathrm{Al}}}{1-n A_{n}}\right) \cdot \rho R T \\
& =A\left(\lambda_{\mathrm{Al}}\right) \cdot \rho R T .
\end{aligned}
$$

Based on the first law of thermodynamics, the equation can be obtained as follows:

$$
C_{p}=C_{v}+\frac{d(P v)}{d T}
$$

where $C_{p}$ is specific heat of products at constant pressure, $C_{v}$ is specific heat of products at constant volume, $P$ is pressure of products, and $v$ is specific volume.

By inserting (4) in (5), it can be found that

$$
C_{p}=C_{v}+\frac{d\left(\left(b+a\left(\lambda_{\mathrm{Al}} /\left(1-n A_{n}\right)\right)\right) \cdot R T\right)}{d T} .
$$

Following the assumption of local isentropic process, the expansion of products in the tube was divided into several small time ranges. The rate of $\mathrm{Al}$ oxidation is slow relative to the short time ranges and the mass fraction of reacted aluminum is approximately constant. In each time range, (6) can be simplified as follows:

$$
C_{p}=C_{v}+\left(b+a \frac{\lambda_{\mathrm{Al} i}}{1-n A_{n}}\right) \cdot R .
$$

The adiabatic index of the products is

$$
\gamma=\frac{C_{p}}{C_{v}} .
$$

By inserting (8) in (7), it can be found that

$$
C_{p}=\frac{\gamma}{\gamma-1}\left(b+a \frac{\lambda_{\mathrm{Al} i}}{1-n A_{n}}\right) \cdot R .
$$

Based on the first law of thermodynamics, the following equation can be obtained:

$$
d h=d e+P d v+v d P=C_{p} d T .
$$

Equation (10) can be simplified to

$$
d S=\frac{d q}{T}=\frac{C_{p} d T}{T}-\frac{v}{T} d P .
$$

The state equation of aluminized explosive products (4) can be inserted into (11), and the following equation can be obtained:

$$
d S=\frac{C_{p} d T}{T}-\frac{\left(b+a\left(\lambda_{\mathrm{Ali}} /\left(1-n A_{n}\right)\right)\right) \cdot R}{P} d P .
$$

By inserting (9) in (12), it can be found that

$$
d S=\frac{C_{p} d T}{T}-\frac{\gamma-1}{\gamma} C_{p} \frac{d P}{P} .
$$

Based on the assumption of local isentropic process, the flow of detonation products is approximately isentropic in each time range. Thus

$$
\frac{T}{P^{(\gamma-1) / \gamma}}=C_{i}
$$

By inserting (4) in (14), it can be found that

$$
\frac{P_{\text {total }}}{\rho^{\gamma}}=\left[\left(b+a \frac{\lambda_{\mathrm{Ali}}}{1-n A_{n}}\right) \cdot C_{i} R\right]^{\gamma},
$$

where $C_{i}$ is constant and $\lambda_{\mathrm{Ali}}$ is the mass fraction of reacted aluminum in time range $i$.

The equations which represent the conservation of mass and momentum behind the detonation front of the aluminized explosive are

$$
\begin{aligned}
& \frac{\partial \rho}{\partial t}+u \frac{\partial \rho}{\partial x}+\rho \frac{\partial u}{\partial x}=0 \\
& \frac{\partial u}{\partial t}+u \frac{\partial u}{\partial x}+\frac{1}{\rho} \frac{\partial p}{\partial x}=0
\end{aligned}
$$




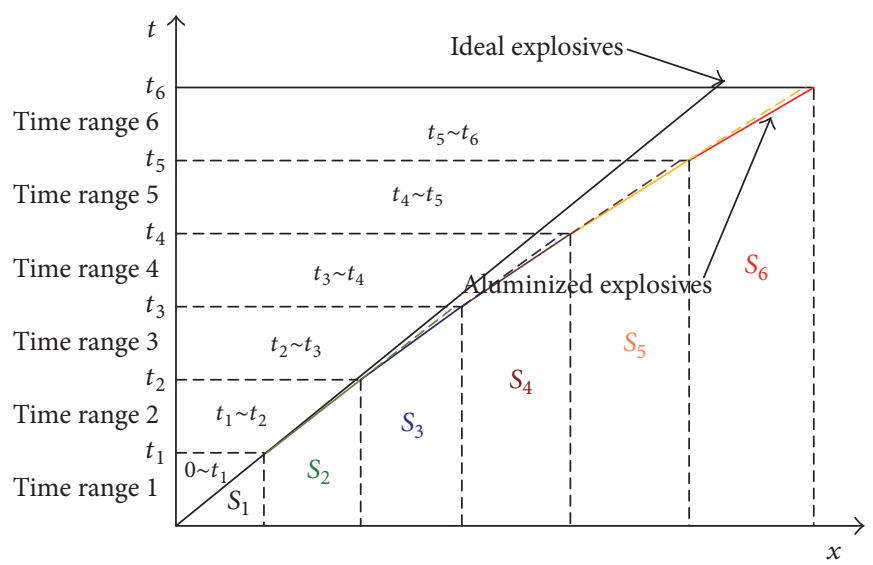

FIGURE 1: Sketch of characteristics for aluminized explosive and ideal explosive.

In each time range, using the method of characteristics for planar isentropic flow, (16) can be converted to ordinary differential equation. In time range $i$, the Riemann invariant along a right-running characteristic is

$$
u_{i}+\frac{2}{\gamma-1} c_{i}=\text { constant. }
$$

When $\gamma=3, u+c=$ constant. Therefore, the rightrunning wave has a constant slope in time range $i$ :

$$
\frac{d x}{d t}=u_{i}+c_{i}
$$

The same can be shown for left-running characteristics. However, the slope of the characteristic is different in every time range due to the reaction of $\mathrm{Al}$ oxidation in the detonation products. Therefore, the characteristics of aluminized explosive and ideal explosive differ, as shown by the sketch in Figure 1.

From the sketch of characteristics for aluminized explosive and ideal explosive (Figure 1), the influence of chemical reaction in the products is apparent. Based on the assumption of local isentropic process, the characteristic for the flow of detonation products of aluminized explosive is divided into several segments along time axis. In each time range, the flow behind the detonation front of aluminized explosive is approximately isentropic. However, the total flow behind the detonation of aluminized explosive is nonisentropic.

For the flow of the detonation products of aluminized explosive, it can be obtained that $S_{1} \neq S_{2} \neq S_{3} \neq S_{4} \neq S_{5} \neq S_{6}$ which is depicted in Figure 1. Parameters of the flow such as sound speed $c$ and pressure of products $p$ are not unchanged along the characteristic line. The parameters of the flow field at time $t_{1}$ will be analyzed first. Because mass is conserved and the volume is unchanged at the time $t_{1}$, the chemical reaction does not influence the density. In this study, it is assumed that the energy released by the chemical reaction mainly influences the pressure and sound speed of the products, while the particle velocity and approximate temperature of the products remain unchanged. According to (4), it can be obtained that

$$
\begin{aligned}
& p_{2}=\frac{A\left(\lambda_{\mathrm{Al} 2}\right)}{A\left(\lambda_{\mathrm{Al} 1}\right)} p_{1}, \\
& \rho_{2}=\rho_{1}, \\
& u_{2}=u_{1}, \\
& c_{2}=\sqrt{\gamma \frac{p_{2}}{\rho_{2}}},
\end{aligned}
$$

where $p, \rho, u$, and $c$ are pressure, density, particle velocity, and sound speed in the products, respectively, and the subscripts represent the time range. Thus, the parameters of detonation products along the characteristic in the time range $i+1$ can be calculated as follows:

$$
\begin{aligned}
& p_{i+1}=\frac{A\left(\lambda_{\mathrm{Al} i+1}\right)}{A\left(\lambda_{\mathrm{Al} i}\right)} p_{i}, \\
& \rho_{i+1}=\rho_{i}, \\
& u_{i+1}=u_{i}, \\
& c_{i+1}=\sqrt{\gamma \frac{p_{i+1}}{\rho_{i+1}}} .
\end{aligned}
$$

Based on the assumption of local isentropic process, the parameters of the flow along the characteristic are only related to the mass fraction of reacted aluminum $\lambda_{\mathrm{Ali}}$. Therefore, the flow field behind the detonation front of aluminized explosive can be calculated analytically when the mass fraction of reacted aluminum is known.

4.1. The Metal Plate Acceleration Test and Mass Fraction of Reacted Aluminum. The metal plate acceleration test was conducted to obtain the acceleration ability of the explosive and the mass fraction of reacted aluminum. The metal plate was driven by two kinds of explosives in this experiment. The explosives and charge size are shown in Table 1. Sample 1 and 
TABLE 1: Components and charge size of the explosive.

\begin{tabular}{lccc}
\hline Sample number & Proportion of components (wt.\%) & Diameter of Al particle $(\mu \mathrm{m})$ & Charge size $(\mathrm{mm})$ \\
\hline 1 & $\mathrm{RDX} / \mathrm{Al} /$ wax 76/20/4 & 5 & $\Phi 40 \times 15$ \\
2 & $\mathrm{RDX} / \mathrm{LiF} /$ wax 76/20/4 & 5 & $\Phi 40 \times 15$ \\
\hline
\end{tabular}

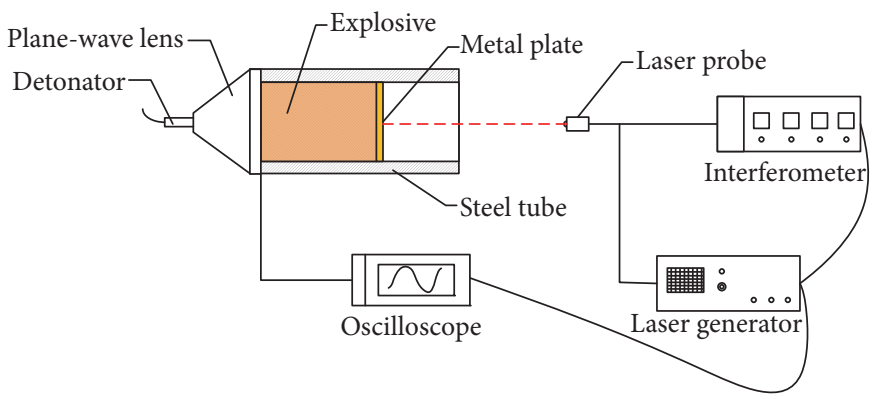

FIGURE 2: Schematic diagram of the metal plate acceleration test.

sample 2 accelerate the same copper plate, which has a charge size of $\Phi 40 \mathrm{~mm} \times 1 \mathrm{~mm}$. The experimental setup is shown in Figure 2.

The reaction time of explosion is on the order of a microsecond, making it difficult to obtain detailed quantitative data of the mass fraction of reacted aluminum directly. Unlike $\mathrm{Al}$ powder, $\mathrm{LiF}$ always remain inert. Based on the test result, the mass fraction of reacted aluminum of detonation products can be obtained indirectly. The difference in kinetic energy of the metal plates driven by $\mathrm{RDX} / \mathrm{Al} /$ wax and $\mathrm{RDX} / \mathrm{LiF} /$ wax is the energy contributed by $\mathrm{Al}$ oxidation in the detonation products. Thus

$$
\sigma Q_{\mathrm{Al}} \omega a \lambda(t)=\frac{1}{2} m\left(V_{\mathrm{Al}}^{2}(t)-V_{\mathrm{LiF}}^{2}(t)\right)
$$

where $\sigma$ is the efficiency of $\mathrm{Al}$ oxidation reaction heat in detonation products, $Q_{\mathrm{Al}}$ is the $\mathrm{Al}$ oxidation reaction heat, $\lambda$ is the mass fraction of reacted aluminum, $\omega$ is the mass of aluminum explosive, $a$ is the mass fraction of aluminum in the explosive, $m$ is the mass of metal plate, and $V_{\mathrm{Al}}$ and $V_{\mathrm{LiF}}$ are the velocities of the metal plates driven by $\mathrm{RDX} / \mathrm{Al} /$ wax (sample 1) and RDX/LiF/wax (sample 2), respectively. The results of metal plate acceleration test are shown in Figure 3.

The reactions of $\mathrm{Al}$ with detonation products mainly contain three parts:

$$
\begin{aligned}
& \mathrm{Al}+1.125 \mathrm{CO} 2 \rightarrow 0.5 \mathrm{Al} 2 \mathrm{O} 3+0.75 \mathrm{CO}+0.37 \mathrm{C} \\
& \triangle \mathrm{H} 1=0.5 \times(-1675.7)+0.75 \times(-110.525)-1.125 \times \\
& (-393.509)=478.046 \mathrm{~kJ} / \mathrm{mol} \\
& \mathrm{Al}+1.5 \mathrm{CO} \rightarrow 0.5 \mathrm{Al} 2 \mathrm{O} 3+1.5 \mathrm{C} \\
& \triangle \mathrm{H} 2=0.5 \times(-1675.7)-1.5 \times(-110.525)= \\
& 672.063 \mathrm{~kJ} / \mathrm{mol} \\
& \mathrm{Al}+1.5 \mathrm{H} 2 \mathrm{O} \rightarrow 0.5 \mathrm{Al} 2 \mathrm{O} 3+1.5 \mathrm{H} 2 \\
& \triangle \mathrm{H} 3=0.5 \times(-1675.7)-1.5 \times(-241.83)= \\
& 475.105 \mathrm{~kJ} / \mathrm{mol} \\
& \mathrm{Q}_{\mathrm{Al}}=(\triangle \mathrm{H} 1+\triangle \mathrm{H} 2+\triangle \mathrm{H} 3) / 27 / 3=20.126 \mathrm{~kJ} / \mathrm{g}
\end{aligned}
$$

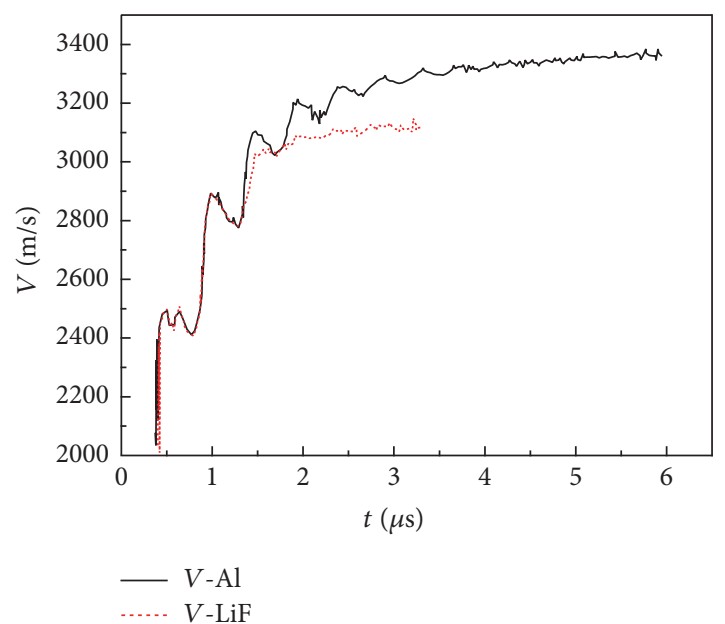

FIGURE 3: Velocity of the metal plate driven by $\mathrm{RDX} / \mathrm{Al} /$ wax and $\mathrm{RDX} / \mathrm{LiF} /$ wax.

When $Q_{\mathrm{Al}}=20.126 \mathrm{~kJ} / \mathrm{g}$ and $\sigma=0.18$ [23], the mass fraction of reacted aluminum in the products can be calculated, as shown in Figure 4.

\section{The Flow of Aluminized Explosive Products behind Metal Plate}

The motion of explosion products is confined to one dimension; thus when the detonation front reaches the metal plate, there will be a reflected compression wave propagating into the products. The nonsimple wave region behind the reflected compression wave and the nonsimple wave region can be represented by the following equations:

$$
\begin{aligned}
& x=\left(u_{i}+c_{i}\right) t+F_{i}, \\
& x=\left(u_{i}-c_{i}\right) t+F_{i}^{\prime},
\end{aligned}
$$




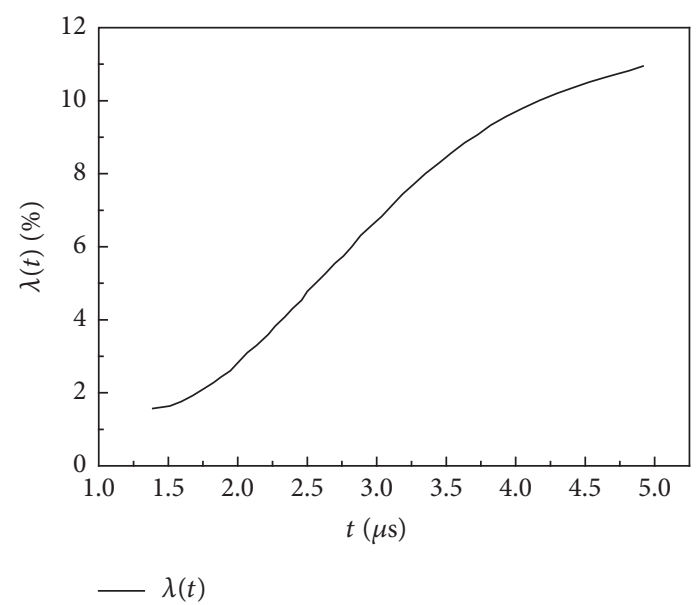

FIgURE 4: The mass fraction of reacted aluminum in detonation products.

where the subscript $i$ represents the flow in the time range $i$. $F_{i}$ is a parameter related to the mass fraction of aluminum $\lambda_{i}$. The mass fraction of aluminum in time range $i$ is treated as approximately constant so $F_{i}$ is also a constant in time range i. $F_{i}^{\prime}$ is also related to the motion of metal plate. Therefore, the motion of metal plate must be calculated first in solving the flow of detonation products.

The equation of conservation of momentum for the metal plate is

$$
M \frac{d V}{d t}=A_{r} p_{m}
$$

where $M$ is the mass of the metal plate, $V$ is the velocity of the metal plate, $A_{r}$ is the surface area of the metal plate, and $p_{m}$ is pressure of detonation products on the surface of metal plate. Considering the equation of the state, the following equation can be obtained:

$$
\frac{p_{m}}{p_{i}}=\left(\frac{c_{m}}{c_{i}}\right)^{\gamma}
$$

where $c_{m}$ is the sound velocity on the surface of the metal plate and $p_{i}$ and $c_{i}$ are the initial condition in the time range $i$.

By inserting (25) in (24), it can be found that

$$
\frac{d V}{d t}=\frac{A_{r} p_{i}}{M c_{i}^{\gamma}} c_{m}^{\gamma}
$$

When the compression waves reach the metal plate, the velocity of detonation products changes from $u_{i}$ to $u_{m}$ which is equal to $V$. The velocity of sound also changes from $c_{i}$ to $c_{m}$. Thus

$$
u_{i}+c_{i}=u_{m}+c_{m}
$$

The derivation of (22), then, is

$$
\frac{d x}{d t}=\left(u_{m}+c_{m}\right)+\left(\frac{d u_{m}}{d t}+\frac{d c_{m}}{d t}\right) t .
$$

By inserting (26) in (28), it can be found that

$$
\frac{d c_{m}}{d t}+\frac{c_{m}}{t}+\psi c_{m}^{\gamma}=0
$$

where $\psi=A_{r} p_{i} / M c_{i}^{\gamma}$.

In this study, $\gamma \approx 3$ and solve the differential equation (29) as follows:

$$
\frac{1+2 \psi c_{m}^{2} t}{c_{m}^{2} t^{2}}=\vartheta_{1}=\text { constant }
$$

In time range 1 , the initial conditions are

$$
\begin{aligned}
& p_{1}=p_{H}=\frac{1}{4} \rho_{0} D^{2}, \\
& c_{1}=c_{H}=\frac{3}{4} D .
\end{aligned}
$$

When the first compression wave reaches the metal plate, the boundary conditions are

$$
\begin{aligned}
t & =\frac{L}{D}, \\
u_{m} & =0, \\
c_{m} & =D,
\end{aligned}
$$

where $\rho_{0}$ is initial density of explosive, $p_{H}$ and $c_{H}$ are $C$ $J$ parameters, $L$ is the length of explosive, and $D$ is the detonation velocity.

By inserting the boundary conditions in (30), it can be found that

$$
\vartheta_{1}=\frac{1+\eta}{L^{2}}=\text { constant }
$$

where $\eta=(32 / 27)(m / M)$.

By inserting (33) in (30), it can be found that

$$
c_{m}=\frac{L}{t} \xi_{1},
$$

where $\xi_{1}=[1+\eta(1-L / D t)]^{-0.5}$.

By inserting (34) in (26) and integrating, it can be found that

$$
V=D\left(1+\frac{2\left(\xi_{1}-1\right)}{\eta \xi_{1}}-\frac{L \xi_{1}}{D t}\right)
$$

The initial and boundary conditions of the time range $i+1$ can be obtained from the properties of the time range $i$. The 
TABLE 2: Characteristics of the explosive and the charge size.

\begin{tabular}{lcccc}
\hline Explosive & Aluminum content $($ wt.\%) & Density $\left(\mathrm{g} \cdot \mathrm{cm}^{-3}\right)$ & Detonation velocity $\left(\mathrm{m} \cdot \mathrm{s}^{-1}\right)$ & Charge size $(\mathrm{mm})$ \\
\hline RDX/Al/wax 76/20/4 & 20 & 1.82 & 8000 & $\Phi 40 \times 15$ \\
RDX/LiF/wax 76/20/4 & - & 1.82 & 8000 & $\Phi 40 \times 15$ \\
\hline
\end{tabular}

velocity of the metal plate driven by aluminized explosive in time range $i+1$ can be calculated:

$$
\begin{aligned}
& V-V_{i}=\left(\frac{A\left(\lambda_{\mathrm{Al} i}\right)}{A\left(\lambda_{\mathrm{Al} 0}\right)}\right)^{1 / 4} \\
& \cdot\left(\frac{L \xi_{i+1}\left(t_{i}\right)}{t_{i}}+\frac{2 D}{\eta} \frac{1}{\xi_{i+1}\left(t_{i}\right)}-\frac{L \xi_{i+1}}{t}-\frac{2 D}{\eta \xi_{i+1}}\right) \\
& \xi_{i+1}=\left(\sqrt{\left.\frac{A\left(\lambda_{\mathrm{Al} i}\right)}{A\left(\lambda_{\mathrm{Al} 0}\right)} L^{2} \vartheta_{i+1}-\frac{\eta L}{D t}\right)^{-0.5}},\right. \\
& \vartheta_{i+1}=\frac{1+\sqrt{A\left(\lambda_{\mathrm{Al0}}\right) / A\left(\lambda_{\mathrm{Ali}}\right)}\left(\eta c_{m}^{2}\left(t_{i}\right) t_{i} / L D\right)}{c_{m}^{2}\left(t_{i}\right) t_{i}^{2}} \\
&=\text { constant, }
\end{aligned}
$$

where $t_{i}$ is the initial time and $V_{i}$ is the initial velocity of the metal plate in the time range $i+1$.

In time range $1\left(t_{0} \sim t_{1}\right)$, the nonsimple wave region behind the metal plate can be represented by the following equations:

$$
\begin{aligned}
& x=\left(u_{1}+c_{1}\right) t+F_{1}, \\
& x=\left(u_{1}-c_{1}\right) t+F_{1}^{\prime},
\end{aligned}
$$

where $F_{1}=0$.

Based on the characteristic method, it can be obtained that

$$
u_{1}+c_{1}=u_{m}+c_{m}
$$

By inserting (34) and (39) in (37), it can be found that

$$
u_{m}=\frac{x-F_{1}}{t}-\frac{L \xi_{1}}{t} .
$$

By subtracting (34) from (40), it can be obtained that

$$
u_{m}-c_{m}=u_{1}-c_{1}=\frac{x-F_{1}}{t}-\frac{2 L \xi_{1}}{t} .
$$

By inserting (41) in (38), it can be found that

$$
F_{1}^{\prime}=F_{1}+2 L \xi_{1}
$$

In time range $2\left(t_{1} \sim t_{2}\right)$, the nonsimple wave region behind the metal plate can be represented by the following equations:

$$
\begin{aligned}
& x=\left(u_{2}+c_{2}\right) t+F_{2}, \\
& x=\left(u_{2}-c_{2}\right) t+F_{2}^{\prime} .
\end{aligned}
$$

As mentioned in the section of the effect of $\mathrm{Al}$ oxidation, the density and velocity of particles along a characteristic remain unchanged. At the time $t_{1}$, the following relationship can be obtained:

$$
x=\left(u_{1}+c_{1}\right) t_{1}=\left(u_{2}+c_{2}\right) t_{1}+F_{2} .
$$

Therefore $F_{2}$ can be obtained:

$$
\begin{aligned}
& F_{2}=\left(1-\sqrt{\frac{A\left(\lambda_{\mathrm{Al} 1}\right)}{A\left(\lambda_{\mathrm{Al} 0}\right)}}\right) c_{1} t_{1}, \\
& F_{2}^{\prime}=F_{2}+2 L \xi_{2} .
\end{aligned}
$$

In time range $i\left(t_{i-1} \sim t_{i}\right)$, the nonsimple wave region behind the metal plate can be represented by the following equations:

$$
\begin{aligned}
x & =\left(u_{i}+c_{i}\right) t+F_{i}, \\
x & =\left(u_{i}-c_{i}\right) t+F_{i}^{\prime}, \\
F_{i} & =\left(1-\sqrt{\frac{A\left(\lambda_{\mathrm{Al} i-1}\right)}{A\left(\lambda_{\mathrm{Al} i-2}\right)}}\right) c_{i-1} t_{i-1}, \\
F_{i}^{\prime} & =F_{i}+2 L \xi_{i} .
\end{aligned}
$$

Therefore, the flow of the aluminized explosive products behind the metal plate can be solved by the described onedimension nonisentropic model.

\section{The Application of the Model to the Aluminized Explosive}

The one-dimension nonisentropic model is applied to calculate the velocity of the metal plate driven by $\mathrm{RDX} / \mathrm{Al} /$ wax (76/20/4) and RDX/LiF/wax (76/20/4). The characteristics of the explosives are shown in Table 2.

The initial and boundary conditions are shown in Table 3.

Based on the assumption of local isentropic process, the process of products expansion was divided into 35 time ranges on average. The velocity of the metal plate and sound speed of the detonation products behind the metal plate were calculated and are depicted in Figure 5.

When calculated by the one-dimension nonisentropic model, the final velocity of the metal plate driven by $\mathrm{RDX} / \mathrm{Al} /$ wax was $3570 \mathrm{~m} / \mathrm{s}$ and the velocity when driven by $\mathrm{RDX} / \mathrm{LiF} /$ wax was $3310 \mathrm{~m} / \mathrm{s}$. The final velocity of the metal plate driven by $\mathrm{RDX} / \mathrm{Al} /$ wax was $7.8 \%$ higher than that of the $\mathrm{RDX} / \mathrm{LiF} /$ wax. When experimentally tested, the final velocity of metal plate driven by $\mathrm{RDX} / \mathrm{Al} /$ wax was $3418 \mathrm{~m} / \mathrm{s}, 9.5 \%$ higher than that of the $\mathrm{RDX} / \mathrm{LiF} / \mathrm{wax}$. The final velocity 
TABLE 3: The initial condition and boundary condition in time range 1.

\begin{tabular}{lcr}
\hline & $p_{H}(\mathrm{GPa})$ & 28.8 \\
Initial condition & $\rho_{H}\left(\mathrm{~g} \cdot \mathrm{cm}^{-3}\right)$ & 2.4 \\
& $u_{H}(\mathrm{~m} / \mathrm{s})$ & 2000 \\
\hline \multirow{2}{*}{ Boundary condition } & $c_{H}(\mathrm{~m} / \mathrm{s})$ & 6000 \\
\hline
\end{tabular}

(a) $p_{H}, \rho_{H}, u_{H}$, and $c_{H}$ represent the pressure, density, velocity of particle in the products, and sound speed on the detonation front.

(b) $u_{m}$ and $c_{m}$ represent the velocity of particle in the products and sound speed behind the metal plate.

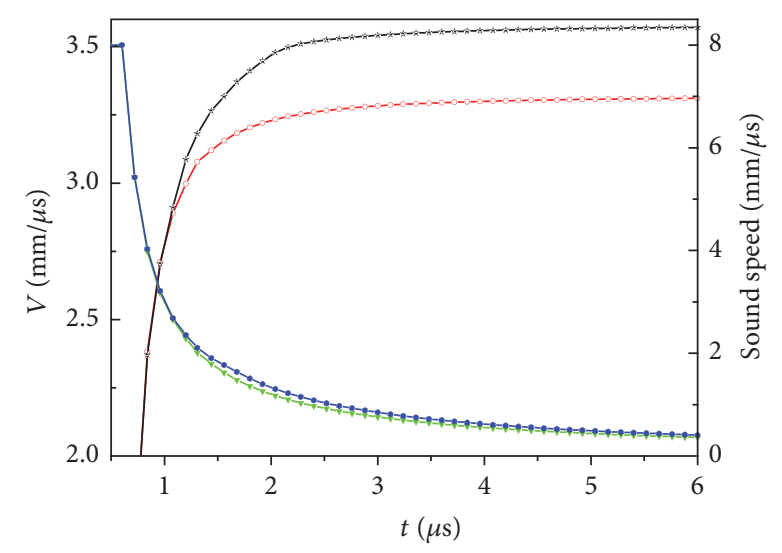

$-V$-RDX/LiF/wax 76/20/4

-* $V$-RDX/Al/wax 76/20/4

$\ldots$ - Sound speed-RDX/LiF/wax

-.- Sound speed-RDX/Al/wax

FIGURE 5: Velocity of metal plate and sound speed of the detonation products behind the metal plate, as calculated by the one-dimension nonisentropic model.

calculated by the model is higher than the experimentally determined velocity (Table 4).

$\mathrm{Al}$ oxidation increases the acceleration ability of the explosive, which can be seen when compared to RDX/ $\mathrm{LiF} /$ wax (Figure 5). The one-dimension nonisentropic model analytically describes the contribution of $\mathrm{Al}$ oxidation in the products. The problem was simplified and the assumption of local isentropic process was employed to analyze the contribution of $\mathrm{Al}$ oxidation in the products. The result calculated by the model cannot be as precise as the result which is calculated by CFD; however the objective of the present study is to develop a simplified analytical model to describe the contribution of $\mathrm{Al}$ oxidation, in the same fashion of the classical isentropic flow model for detonation products of ideal explosive.

Note that the model will produce large error when the interval of time range is divided too largely. We divided the process of detonation products expanding into 10, 15, 20 , and 25 time ranges averagely and calculated the metal plate velocity, respectively. When the process is divided into 20,25 , and 35 time ranges averagely, the velocities of metal plate are almost the same. But the results on 10 and 15 time ranges are not good. If each time range is too large, the mass
TABLE 4: Comparison of the final velocities determined experimentally and through the model.

\begin{tabular}{lcc}
\hline Explosive & \multicolumn{2}{c}{ Final velocity $(\mathrm{m} / \mathrm{s})$} \\
& Experimental & Model \\
\hline $\mathrm{RDX} / \mathrm{Al} /$ wax & 3418 & 3570 \\
$\mathrm{RDX} / \mathrm{LiF} /$ wax & 3121 & 3310 \\
\hline
\end{tabular}

fraction of reacted aluminum cannot be treated as constant approximately and the result calculated by our model will produce large error.

\section{Summary}

In this study, a simple model for aluminized explosive products was constructed and used to calculate the motion of a metal plate driven by aluminized explosive. This model operates on the assumption that the $\mathrm{Al}$ particles have no effect on the flow field before Al particles oxidation. This one-way coupling assumption is valid for small particle loading in the high explosive. The detonation of aluminized explosive contained in a tube is strong enough to conifer the motion to one dimension. It also assumes that the process of products expanding in the tube is adiabatic. In order to solve the complete flow field analytically, the expansion process was divided into several time ranges. In the short time ranges, we assume that the process is approximately isentropic. Based on the assumptions, the model can be solved analytically. The metal plate acceleration test was conducted to indirectly obtain the velocity of metal plate and the mass fraction of reacted aluminum of detonation products. Finally, the model was applied to calculate the motion of the metal plate, and the model and experimental results were compared. The analytic model result was slightly higher than the test result. The model was a simple method that captured the contribution of the $\mathrm{Al}$ oxidation in the products to acceleration ability. Moreover, the flow of aluminized explosive products behind the metal plate can be solved based on the model. The application of the analytic model for the detonation products of aluminized explosive is analogous to the classical isentropic flow model for detonation products of ideal explosives.

\section{Conflicts of Interest}

The authors declare that they have no conflicts of interest. 


\section{References}

[1] P. J. Haskins, M. D. Cook, and R. I. Briggs, "The effect of additives on the detonation characteristics of a liquid explosive," in Proceedings of the 12th APS Topical Group Meeting on Shock Compression of Condensed Matter, pp. 890-893, Atlanta, Ga, USA, June 2001.

[2] D. L. Frost, S. Goroshin, R. Ripley, and F. Zhang, "Effect of scale on the blast wave from a metalized explosive," in Proceedings of the 13th International Detonation Symposium, Norfolk, Va, USA, July 2006.

[3] D. L. Frost, S. Goroshin, J. Levine, R. Ripley, and F. Zhang, "Critical conditions for ignition of aluminum particles in cylindrical explosive charges," in Proceedings of the Conference of the American Physical Society Topical Group on Shock Compression of Condensed Matter (AIP '05), pp. 972-975, Baltimore, Md, USA, 2005.

[4] F. Zhang, A. Yoshinaka, D. Frost, R. Ripley, K. Kim, and W. Wilson, "Casing influence on ignition and reaction of aluminum particles in an explosive," in Proceedings of the 13th International Detonation Symposium, Norfolk, Va, USA, July 2006.

[5] R. C. Ripley, L. Donahue, T. E. Dunbar, and F. Zhang, "Explosion performance of aluminized TNT in a chamber," in Proceedings of the 19th International Symposium on Military Aspects of Blast and Shock, Calgary, Canada, October 2006.

[6] A. M. Milne, S. Cargill, K. Bennett, and A. W. Longbottom, "Modelling of enhanced blast and heterogeneous explosives," in Proceedings of the 19th International Symposium on Military Aspects of Blast and Shock, Alberta, Canada, October 2006.

[7] M. A. Cooper, M. R. Baer, R. G. Schmitt, M. J. Kaneshige, R. J. Pahl, and P. E. DesJardin, "Understanding enhanced blast explosives: a multiscale challenge," in Proceedings of the 19th International Symposium on Military Aspects of Blast and Shock, Calgary, Canada, October 2006.

[8] J. Massoni, R. Saurel, A. Lefrançois, and G. Baudin, "Modeling spherical explosions with aluminized energetic materials," Shock Waves, vol. 16, no. 1, pp. 75-92, 2006.

[9] K. Kim, W. Wilson, S. Peiris et al., "Effects of particle damage during detonation of thermobarics on subsequent reactions," in Proceedings of the 21st International Colloquium, on the Dynamics of Explosions and Reactive Systems, Poitiers, France, July 2007.

[10] D. L. Frost, T. Aslam, and L. G. Hill, "Application of detonation shock dynamics to the propagation of a detonation in nitromethane in a packed inert particle bed," in AIP Conference Proceedings-Shock Compression of Condensed Matter, pp. 821824, 2000.

[11] R. C. Ripley, F. Zhang, and F.-S. Lien, "Detonation interaction with metal particles in explosives," in Proceedings of the 13th International Detonation Symposium (IDS '06), pp. 214-223, Norfolk, Va, USA, July 2006.

[12] F. Zhang, P. A. Thibault, and R. Link, "Shock interaction with solid particles in condensed matter and related momentum transfer," Proceedings of the Royal Society of London A: Mathematical, Physical and Engineering Sciences, vol. 459, no. 2031, pp. 705-726, 2003.

[13] K. Seshadri, A. L. Berlad, and V. Tangirala, "The structure of premixed particle-cloud flames," Combustion and Flame, vol. 89, no. 3-4, pp. 333-342, 1992.

[14] G. Taylor, "The dynamics of the combustion products behind plane and spherical detonation fronts in explosives," Proceedings of the Royal Society of London A: Mathematical, Physical and Engineering Sciences, vol. 200, no. 1061, pp. 235-247, 1950.

[15] K. P. Stanyukovich, Unsteady Motion of Continuous Media, Pergamon Press, London, UK, 1960.

[16] P. J. Miller, "A reactive flow model with coupled reaction kinetics for detonation and combustion in non-ideal explosives," in Proceedings of the Materials Research Society Symposium, pp. 413-420, Materials Research Society (MRS), Boston, Mass, USA, November 1995.

[17] Z. Q. Zhou, J. X. Nie, Z. C. Ou, J. F. Qin, and Q. J. Jiao, "Effects of the aluminum content on the shock wave pressure and the acceleration ability of RDX-based aluminized explosives," Journal of Applied Physics, vol. 116, no. 14, Article ID 144906, 2014.

[18] P. J. Miller and R. H. Guirguis, "Effects of late chemical reactions on the energy partition in non-ideal underwater explosions," in Proceedings of the Joint AIRAPT/APS Conference on High Pressure Science and Technology, p. 1417, Colorado Springs, Colo, USA, June 1993.

[19] P. J. Miller, Reaction Kinetics of Aluminum Particles in Detonation Gases, vol. 615 of JANNAF PSHS Proceedings, CPIA Publication, San Diego, Calif, USA, 1994.

[20] G. Rudinger, Fundamentals of Gas-Particle Flow, Elsevier, Amsterdam, The Netherlands, 1980.

[21] K. Balakrishnan, A. L. Kuhl, J. B. Bell, and V. E. Beckner, "Ignition of aluminum particle clouds behind reflected shock waves," in Proceedings of the 23rd International Colloquium on the Dynamics of Explosions and Reactive Systems (ICDERS '11), Irvine, Calif, USA, 2011.

[22] B. Kim, J. Park, K.-C. Lee, and J. J. Yoh, "A reactive flow model for heavily aluminized cyclotrimethylene-trinitramine," Journal of Applied Physics, vol. 116, no. 2, Article ID 023512, 2014.

[23] L. Chen, X. Long, C. Feng, and X. Jiang, The Detonation of Aluminized Explosive, National Defense Industry Press, Beijing, China, 2004. 


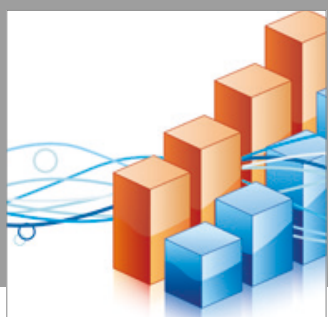

Advances in

Operations Research

vatersals

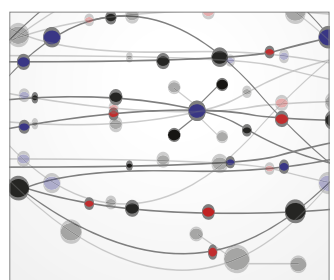

\section{The Scientific} World Journal
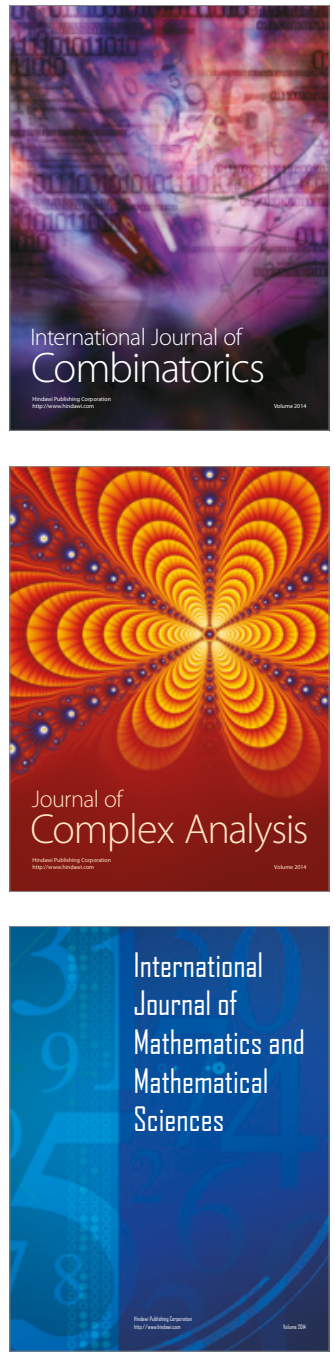
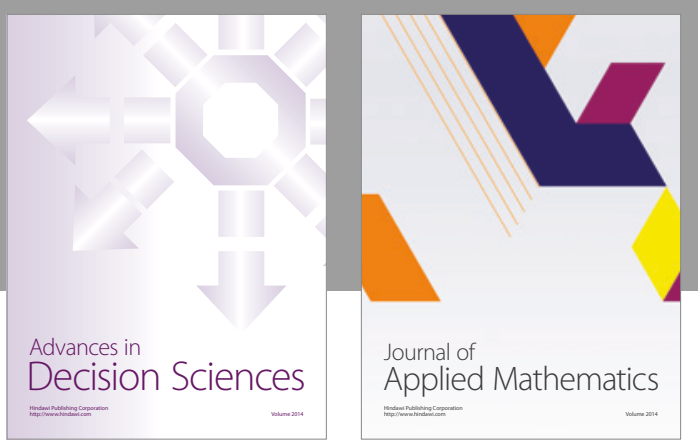

Algebra

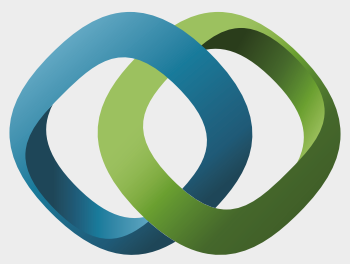

\section{Hindawi}

Submit your manuscripts at

https://www.hindawi.com
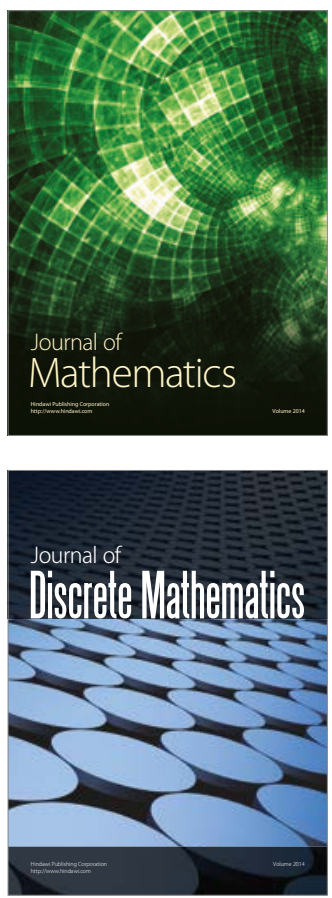

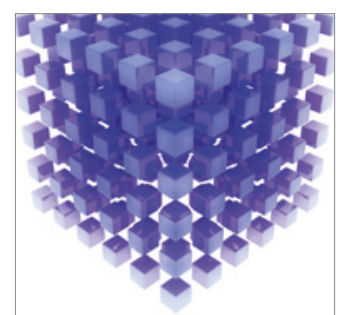

Mathematical Problems in Engineering
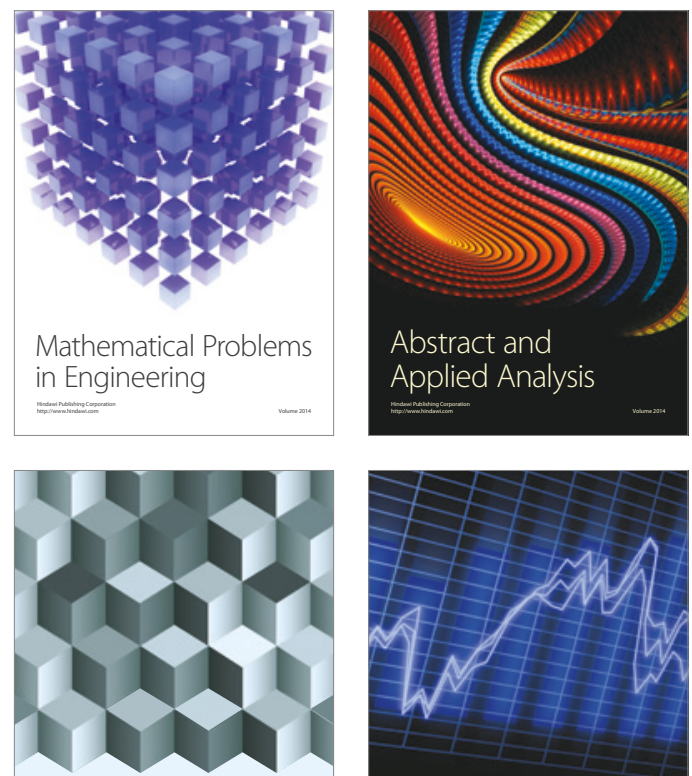

Journal of

Function Spaces

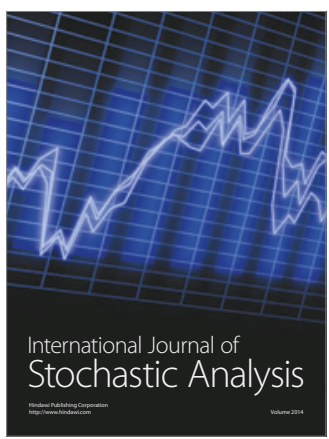

Probability and Statistics
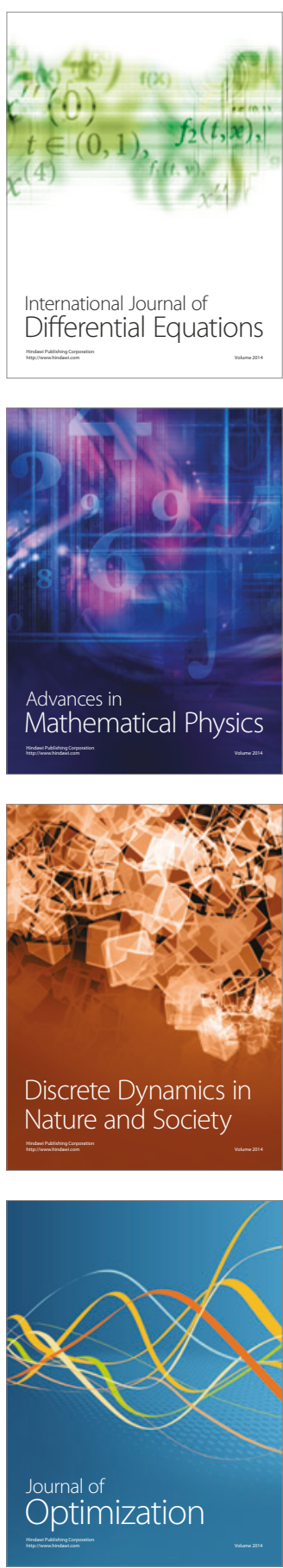